\title{
Experimental study of water jet break-up in subcritical and supercritical
}

\section{carbon dioxide}

Paolo Trucilloa, Roberta Campardellia,b, lolanda De Marco ${ }^{a, *}$

a Department of Industrial Engineering (DIIN), University of Salerno, Via Giovanni Paolo II, 132 84084 Fisciano (SA), ITALY

${ }^{b}$ Department of Civil, Chemical and Environmental Engineering (DICCA), University of Genoa, Via Opera Pia 15, 16145 Genova (GE), ITALY

*idemarco@unisa.it 
Table S1. Calculation of the characteristic jet parameter using previous existing methods (WFR $1 \mathrm{~mL} / \mathrm{min}$ )

\begin{tabular}{|c|c|c|c|c|c|c|}
\hline \multicolumn{2}{|c|}{ Models } & $\begin{array}{c}\text { Reitz \& Bracco } \\
(\mathbf{1 9 8 6})^{\mathbf{1}}\end{array}$ & $\begin{array}{c}\text { Chatwani \& } \\
\text { Bracco (1985) }\end{array}$ & $\begin{array}{c}\text { Reitz \& Bracco } \\
(\mathbf{1 9 8 6})^{\mathbf{1}}\end{array}$ & $\begin{array}{c}\text { Reitz \& } \\
\text { Diwakar } \\
(\mathbf{1 9 8 7})^{\mathbf{3}}\end{array}$ & $\begin{array}{c}\text { Reitz } \\
(\mathbf{1 9 8 7})^{4}\end{array}$ \\
\hline $\mathbf{P}$ & $\mathbf{T}$ & $\mathbf{L}_{\mathbf{2}}$ & $\mathbf{L}_{\mathbf{1}}(\mathbf{C}=\mathbf{7 . 1 5})$ & $\boldsymbol{\theta / 2}$ & $\boldsymbol{\theta / 2}$ & $\boldsymbol{\theta / 2}$ \\
\hline$[\mathrm{bar}]$ & {$\left[{ }^{\circ} \mathbf{C}\right]$} & {$[\boldsymbol{\mu m}]$} & {$[\boldsymbol{\mu m}]$} & {$\left[{ }^{\circ}\right]$} & {$\left[{ }^{\circ}\right]$} & {$\left[{ }^{\circ}\right]$} \\
\hline 50 & 40 & 3014 & 3014 & 13.807 & 13.285 & 14.808 \\
\hline 50 & 60 & 446 & 3187 & 13.083 & 12.462 & 14.727 \\
\hline 75 & 40 & 299 & 2137 & 19.118 & 18.719 & 18.242 \\
\hline 75 & 60 & 340 & 2433 & 16.932 & 16.288 & 17.030 \\
\hline 100 & 40 & 187 & 1337 & 28.985 & 28.976 & 26.259 \\
\hline 100 & 60 & 268 & 1914 & 21.150 & 20.598 & 19.996 \\
\hline 125 & 40 & 173 & 1240 & 30.850 & 30.956 & 27.829 \\
\hline 125 & 60 & 210 & 1501 & 26.259 & 25.843 & 24.154 \\
\hline 150 & 40 & 169 & 1205 & 31.579 & 31.749 & 28.427 \\
\hline 150 & 60 & 187 & 1336 & 28.994 & 28.671 & 26.472 \\
\hline
\end{tabular}

Table S2. Calculation of the characteristic jet parameter using previous existing methods (WFR $\mathbf{5} \mathbf{~ m L} / \mathbf{m i n}$ )

\begin{tabular}{|c|c|c|c|c|c|c|}
\hline \multicolumn{2}{|c|}{ Models } & $\begin{array}{c}\text { Reitz \& Bracco } \\
(\mathbf{1 9 8 6})^{\mathbf{1}}\end{array}$ & $\begin{array}{c}\text { Chatwani \& } \\
\text { Bracco (1985) }\end{array}$ & $\begin{array}{c}\text { Reitz \& Bracco } \\
(\mathbf{1 9 8 6})^{1}\end{array}$ & $\begin{array}{c}\text { Reitz \& } \\
\text { Diwakar } \\
(\mathbf{1 9 8 7})^{3}\end{array}$ & $\begin{array}{c}\text { Reitz } \\
(\mathbf{1 9 8 7})^{4}\end{array}$ \\
\hline $\mathbf{P}$ & $\mathbf{T}$ & $\mathbf{L}_{\mathbf{2}}$ & $\mathbf{L}_{\mathbf{1}}(\mathbf{C}=\mathbf{7 . 1 5})$ & $\boldsymbol{\theta / 2}$ & $\boldsymbol{\theta / 2}$ & $\boldsymbol{\theta / 2}$ \\
\hline$[\mathbf{b a r}]$ & {$\left[{ }^{\circ} \mathbf{C}\right]$} & {$[\boldsymbol{\mu m}]$} & {$[\boldsymbol{\mu m}]$} & {$\left[{ }^{\circ}\right]$} & {$\left[{ }^{\circ}\right]$} & {$\left[{ }^{\circ}\right]$} \\
\hline 50 & 40 & 447 & 3194 & 13.054 & 13.285 & 11.300 \\
\hline 50 & 60 & 466 & 3333 & 12.528 & 12.462 & 11.038 \\
\hline 75 & 40 & 324 & 2314 & 17.745 & 18.719 & 14.950 \\
\hline 75 & 60 & 360 & 2575 & 16.046 & 16.288 & 13.867 \\
\hline 100 & 40 & 208 & 1487 & 26.470 & 28.976 & 21.962 \\
\hline 100 & 60 & 288 & 2058 & 19.794 & 20.598 & 16.801 \\
\hline 125 & 40 & 194 & 1387 & 28.106 & 30.956 & 23.293 \\
\hline 125 & 60 & 229 & 1635 & 24.372 & 25.843 & 20.491 \\
\hline 150 & 40 & 189 & 1351 & 28.725 & 31.749 & 23.787 \\
\hline 150 & 60 & 205 & 1464 & 26.831 & 28.671 & 22.502 \\
\hline
\end{tabular}


Table S3. Calculation of the characteristic jet parameter using previous existing methods (WFR $10 \mathrm{~mL} / \mathrm{min}$ )

\begin{tabular}{|c|c|c|c|c|c|c|}
\hline \multicolumn{2}{|c|}{ Models } & $\begin{array}{c}\text { Reitz \& Bracco } \\
(\mathbf{1 9 8 6})^{\mathbf{1}}\end{array}$ & $\begin{array}{c}\text { Chatwani \& } \\
\text { Bracco (1985) }\end{array}$ & $\begin{array}{c}\text { Reitz \& Bracco } \\
(\mathbf{1 9 8 6})^{\mathbf{1}}\end{array}$ & $\begin{array}{c}\text { Reitz \& } \\
\text { Diwakar } \\
(\mathbf{1 9 8 7})^{\mathbf{3}}\end{array}$ & $\begin{array}{c}\text { Reitz } \\
(\mathbf{1 9 8 7})^{4}\end{array}$ \\
\hline $\mathbf{P}$ & $\mathbf{T}$ & $\mathbf{L}_{\mathbf{2}}$ & $\mathbf{L}_{\mathbf{1}}(\mathbf{C = 7 . 1 5})$ & $\boldsymbol{\theta / 2}$ & $\boldsymbol{\theta} / \mathbf{2}$ & $\boldsymbol{\theta / 2}$ \\
\hline$[\mathrm{bar}]$ & {$\left[{ }^{\circ} \mathbf{C}\right]$} & {$[\boldsymbol{\mu m}]$} & {$[\boldsymbol{\mu m}]$} & {$\left[{ }^{\circ}\right]$} & {$\left[{ }^{\circ}\right]$} & {$\left[{ }^{\circ}\right]$} \\
\hline 50 & 40 & 467 & 3337 & 12.514 & 13.285 & 10.435 \\
\hline 50 & 60 & 482 & 3448 & 12.121 & 12.462 & 10.305 \\
\hline 75 & 40 & 343 & 2454 & 16.792 & 18.719 & 13.661 \\
\hline 75 & 60 & 376 & 2687 & 15.408 & 16.288 & 12.850 \\
\hline 100 & 40 & 224 & 1605 & 24.771 & 28.976 & 19.977 \\
\hline 100 & 60 & 304 & 2171 & 18.839 & 20.598 & 15.437 \\
\hline 125 & 40 & 210 & 1501 & 26.258 & 30.956 & 21.188 \\
\hline 125 & 60 & 243 & 1740 & 23.061 & 25.843 & 18.749 \\
\hline 150 & 40 & 205 & 1466 & 26.809 & 31.749 & 21.636 \\
\hline 150 & 60 & 219 & 1564 & 25.334 & 28.671 & 20.572 \\
\hline
\end{tabular}

Table S4. Calculation of the characteristic jet parameter using previous existing methods (WFR $15 \mathrm{~mL} / \mathrm{min}$ )

\begin{tabular}{|c|c|c|c|c|c|c|}
\hline \multicolumn{2}{|c|}{ Models } & $\begin{array}{c}\text { Reitz \& Bracco } \\
(\mathbf{1 9 8 6})^{\mathbf{1}}\end{array}$ & $\begin{array}{c}\text { Chatwani \& } \\
\text { Bracco (1985) }\end{array}$ & $\begin{array}{c}\text { Reitz \& Bracco } \\
(\mathbf{1 9 8 6})^{\mathbf{1}}\end{array}$ & $\begin{array}{c}\text { Reitz \& } \\
\text { Diwakar } \\
(\mathbf{1 9 8 7})^{\mathbf{3}}\end{array}$ & $\begin{array}{c}\text { Reitz } \\
(\mathbf{1 9 8 7})^{4}\end{array}$ \\
\hline $\mathbf{P}$ & $\mathbf{T}$ & $\mathbf{L}_{\mathbf{2}}$ & $\mathbf{L}_{\mathbf{1}}(\mathbf{C = 7 . 1 5})$ & $\boldsymbol{\theta / 2}$ & $\boldsymbol{\theta} / \mathbf{2}$ & $\boldsymbol{\theta / 2}$ \\
\hline$[\mathrm{bar}]$ & {$\left[{ }^{\circ} \mathbf{C}\right]$} & {$[\boldsymbol{\mu m}]$} & {$[\boldsymbol{\mu m}]$} & {$\left[{ }^{\circ}\right]$} & {$\left[{ }^{\circ}\right]$} & {$\left[{ }^{\circ}\right]$} \\
\hline 50 & 40 & 482 & 3449 & 12.121 & 13.285 & 9.898 \\
\hline 50 & 60 & 495 & 3539 & 11.819 & 12.462 & 9.840 \\
\hline 75 & 40 & 359 & 2564 & 16.114 & 18.719 & 12.887 \\
\hline 75 & 60 & 388 & 2776 & 14.940 & 16.288 & 12.205 \\
\hline 100 & 40 & 237 & 1697 & 23.582 & 28.976 & 18.843 \\
\hline 100 & 60 & 316 & 2259 & 18.151 & 20.598 & 14.597 \\
\hline 125 & 40 & 222 & 1591 & 24.968 & 30.956 & 19.997 \\
\hline 125 & 60 & 255 & 1822 & 22.125 & 25.843 & 17.705 \\
\hline 150 & 40 & 217 & 1555 & 25.473 & 31.749 & 20.425 \\
\hline 150 & 60 & 230 & 1643 & 24.268 & 28.671 & 19.426 \\
\hline
\end{tabular}

(1) Reitz, R. D.; Bracco, F., Mechanism of breakup of round liquid jets. In Encyclopedia of fluid mechanics, Cheremisnoff, N., Ed. Gulf Publishing: Houston, Texas, 1986; pp 223-249.

(2) Chatwani, A.; Bracco, F., Computation of dense spray jets. In Proceedings of the 3rd International Conference on Liquid Atomisation and Spray Systems, London, England, 8-10 July, 1985.

(3) Reitz, R. D.; Diwakar, R., Structure of high-pressure fuel sprays. SAE transactions 1987, 96, 492.

(4) Reitz, R., Modeling atomization processes in high-pressure vaporizing sprays. In Atomisation and Spray Technology, 1987; Vol. 3, pp 309-337. 